\title{
ANÁLISIS DE LA ASOCIACIÓN ENTRE LA CALIDAD VIDA Y LA CONDICIÓN ORAL DE LAS PERSONAS MAYORES VINCULADAS A TRES INSTITUCIONES GERIÁTRICAS DE BUCARAMANGA: II FASE *
}

\author{
${ }^{1}$ Sonia Constanza Concha Sánchez, ${ }^{2}$ Diana Marina Camargo Lemus \\ ${ }^{1}$ Odontóloga U. Santo Tomás, Especialista en Educación y Comunicación para la Salud, \\ MSc Epidemiología U. Industrial de Santander, Docente U. Santo Tomás \\ ${ }^{2}$ Bacteriologa Universidad Javeriana, Especialista en Docencia Universitaria, \\ M.Sc. Epidemiología, Docente U. Industrial de Santander
}

Autor responsable de correspondencia: Sonia Constanza Concha Sánchez

Dirección de correo electrónico: sococosa@yahoo.com

\section{RESUMEN}

Objetivo: Evaluar la asociación entre la calidad de vida (CV) con la condición oral (CO) en las personas mayores.

Materiales y métodos: Se realizó un estudio observacional analítico de corte transversal. El universo constituido por 523 y la muestra por 106 personas de tres instituciones geriátricas de la ciudad, que cumplieron con los criterios de inclusión. Se estudiaron variables sociodemográficas; condiciones sistémicas, la CV, la CO, la capacidad física funcional la condición mental y los soportes sociales. Para el análisis se calcularon medidas de resumen según la naturaleza de cada variable. Se realizó un análisis de factores para determinar los atributos que integraron el constructo de CO. Se relacionó la variable CV con cada variable explicatoria mediante pruebas de $\chi^{2}$ y test exacto de Fisher. Se calcularon los OR crudos y sus intervalos de confianza. Se aplicó análisis de regresión logística, métodos de corrección de los OR, obteniendo los RR y se evalúo la bondad de ajuste del modelo.

Resultados: La CV en las personas mayores registró un promedio de 49.9 18.6 con rango entre 14 y 85 . El índice COP, el flujo salival y el dolor oral fueron atributos que definieron la CO; el $72 \%$ de la población tenía una mala CO. No se logró establecer ninguna asociación entre una mala CO con una mala CV en las personas mayores analizadas, con RR 0.99 (IC95\% 0.56-1.41).

Conclusión: Una mala CO no parece estar asociada con una mala CV en las personas mayores analizadas; estos resultados parecen indicar la poca ingerencia que tiene una mala condición oral, condición frecuente en la población evaluada, relación podría estar afectada por aspectos culturales que hacen que la sociedad en general y el adulto mayor en particular, pueda llegar a subestimar esta condición. [Concha SC, Camargo DM. Análisis de la asociación entre la calidad vida y la condición oral de las personas mayores vinculadas a tres instituciones geriátricas de Bucaramanga: II fase. Revista Ustasalud Odontología 2007; 6: 75 - 86]

Palabras clave: Calidad de Vida, Adulto Mayor, Salud Oral

\section{EVALUATION OF THE ASSOCIATION BETWEEN ORAL CONDITIONS WITH QUALITY OF LIFE IN THE OLDEST WHOM LIVED IN THREE ELDERLY INSTITUTION OF BUCARAMANGA}

\begin{abstract}
Objective: The aim was to evaluate the association between the quality of life (QL) with the oral condition (OC) in the elderly. Material and methods: An analytical cross section observational study was made. The universe was constituted by 523 and the sample by 106 people, who lived in three elderly institution of the city, who fulfilled the inclusion criteria. The variables of the study were social-demographic; systemic condition, the QL, the OC, the functional physical capacity, mental state, and the social supports In the analysis, summary measures were calculated according to the nature of each variable. Analysis of factors was made to determine the attributes that integrated OC. The exit variable (QL) was related to each other variables by $\chi^{2}$ and Fisher's exact test, the crude OR and intervals of confidence were calculated. Logistic regression analysis and methods of correction of the OR were applied, the RR were obtained and the goodness of fit of the model was evaluated.

Results: The quality of life in the elderly registered an average of $49.9 \pm 18.6$ with rank between 14 and 85 . The COP index, the salivary flow and the oral pain were attributes that defined the oral condition; the $72 \%$ of the population had a bad oral condition. A potential association between bad OC with bad QL of the elderly wasn't seen RR 0.99 (IC95\% 0.56-1.41).

Conclusion: A bad CO does not seem to be associate with bad a QL in the elderly; these results seem to indicate the little effect that has a bad oral condition, frequent condition in the evaluated population, relation could be affected by cultural aspects that do that the society in general and the greater adult in individual, can get to underestimate this condition
\end{abstract}

Key words: Quality of Life, Elderly, Oral Health

Recibido para publicación: 24 de noviembre de 2007. Aceptado para publicación: 2 de febrero de 2008.

* Grupo de Investigación SIBAM. 


\section{INTRODUCCIÓN}

La pirámide poblacional en el mundo está cambiando y se observa que la especie humana está envejeciendo. Las proyecciones de la ONU indican que el mayor aumento se dará en los países en vías de desarrollo durante la primera mitad del siglo XXI. Esta transformación demográfica en los ámbitos nacional e internacional, tiene profundas consecuencias en todos los aspectos de la vida individual y comunitaria, como los sociales, físicos, económicos, políticos, culturales, psicológicos y espirituales. ${ }^{1}$

En países en vías de desarrollo, se plantea un importante desafio, pues mientras los países desarrollados han podido envejecer gradualmente, los llamados países del tercer mundo, afrontan un reto simultáneo de desarrollo y de envejecimiento ${ }^{1}$. En Colombia la situación es aún más crítica, pues se ve enfrentada a una mortalidad y emigración de adultos jóvenes muy alta (correspondiente al $8.4 \%$ de la estructura total de la población emigrante) ${ }^{2}$ como consecuencia de la violencia, que sumado a la reducción en los nacimientos, incrementará la proporción de personas ancianas vulnerables, en especial mujeres. ${ }^{3}$

El reto al que se enfrentan las ciencias de la salud con sus avances científicos, al igual sus profesionales, es brindar oportunidades que permitan a los adultos mayores alcanzar y/o conservar una mejor calidad de vida, puesto que cantidad no necesariamente implica calidad de vida; ${ }^{4}$ si bien es cierto, que a medida que transcurren los años en el organismo de cada ser humano ocurren una serie de cambios relacionados con el ciclo de vida, dichas modificaciones no son iguales en todas las personas, ni en todas sus estructuras y no se ha delimitado en forma precisa que es lo normal y lo anormal dentro de este proceso. ${ }^{5,6}$

El concepto de calidad de vida ha venido cobrando importancia, ya que resume en un solo indicador, un constructo multidimensional, que describe la condición de un individuo. ${ }^{4,7,8} \mathrm{El}$ término "calidad de vida" ha tenido múltiples interpretaciones, desde las simplemente hedonistas hasta las relacionadas con los ideales de una buena vida. Debe evaluarse en términos de la capacidad para satisfacer las metas y los objetivos propuestos, que representan parte del desarrollo de una persona, en particular, las cosas que logra hacer o ser al vivir. Sin embargo, no excluye el cubrir necesidades elementales como estar bien nutrido, ser querido, tener la libertad de elegir o tener buena salud; lo cual implica evaluar desde los aspectos más esenciales hasta los más complejos.?
Con el fundamento anterior, es prioritario puntualizar que la relación entre la calidad de vida y salud va más allá de las manifestaciones directas de dolor y de morbilidad; las investigaciones han mostrado la necesidad de elaborar un modelo con base en un constructo multifactorial con componentes consistentes, concepto que emerge como un intento de analizar en términos científicos y cuantificables las consecuencias de la enfermedad y el tratamiento; su impacto sobre la vida, teniendo en cuenta la percepción de la persona y su habilidad para vivir plenamente. ${ }^{7,8}$

Saber cuáles atributos se deben evaluar es motivo de controversia, la mayoría de los autores coinciden en involucrar al menos cuatro dimensiones con distintas representaciones y estas son: el estado fisico y las habilidades funcionales, el estado psicológico y de bienestar; las interacciones sociales y las sensaciones somáticas. $^{8}$

Dentro de las habilidades funcionales, la boca desempeña tanto actividades biológicas y sicológicas como sociales; por lo que la alteración en su condición, puede comprometer la calidad de vida de los individuos. La preservación, recuperación y mantenimiento de la salud y la funcionalidad de la cavidad bucal, en el adulto mayor, repercute de manera significativa en su calidad de vida. Sin embargo, la responsabilidad de esta labor no recae exclusivamente sobre el odontólogo, sino en todos los profesionales de la salud o de las humanidades; en el Estado y en su definición de políticas claras para la protección integral de las personas mayores y en la sociedad en general, particularmente la familia. ${ }^{8}$

Hasta el momento varias publicaciones en Colombia determinan la condición oral del adulto mayor en diferentes indicadores. ${ }^{9-13}$ Algunas incluso abordan teóricamente la relación de salud oral y calidad de vida. ${ }^{9}$ Sin embargo, no hay ninguna que establezca objetiva e integralmente esta relación; aspecto que ha sido demostrado en otras publicaciones y que señala con claridad el impacto negativo que tiene una condición oral inadecuada sobre la calidad de vida. ${ }^{14}$

Un estudio realizado por Strauss y Hunt, muestra cómo una buena salud bucal influye en forma positiva en aspectos como la apariencia, la alimentación, la sensación de confort, la longevidad, la autoconfian$\mathrm{za}$, el habla, el disfrute de la vida, la salud general, el sonreír y reír, la selección de alimentos y la felicidad en general..$^{9.14}$

De otro lado, es importante mencionar que la salud oral es un derecho y una oportunidad para todas las personas, del que no se pueden aislar a las personas 
mayores, habitualmente mantenidas al margen de los programas de bienestar y asistencia social de los gobiernos; ${ }^{10}$ con esto en mente, los profesionales de la odontología no pueden olvidar el compromiso ético de brindar una atención integral, que permita a las personas mayores obtener una buena salud oral y con ello mejores alternativas para alcanzar una mejor calidad de vida.

Lo expuesto anteriormente llevó a la realización de este trabajo, cuyo objetivo fue analizar la asociación entre la condición oral con la calidad de las personas adultas mayores vinculadas a tres instituciones geriátricas de Bucaramanga

\section{MATERIALES Y MÉTODOS}

Se realizó un estudio observacional analítico de corte transversal. ${ }^{15,16}$ La población de estudio estuvo conformada por la 523 individuos, y la muestra por 106 personas vinculadas a tres instituciones geriátricas de Bucaramanga. La recolección de los datos se realizó entre Septiembre de 2006 y Marzo de 2007.

Como criterios de inclusión se consideraron ser adulto mayor residente en las instituciones participantes; no se incluyeron a personas con diagnóstico de patologías psiquiátricas mayores, hipoacusia marcada y con enfermedades severas.

Las variables se registraron en un instrumento diseñado para este fin; la variable de salida fue calidad de vida: En la que se interrogó al paciente sobre su autopercepción en cuanto a su calidad de vida; se recolectó mediante una escala análoga visual. ${ }^{17} \mathrm{con}$ puntajes entre 0 y 100 y se dicotomizó con base en la mediana (52 $\mathrm{mm}$ ), donde los puntajes mayores de 52 representaban una buena calidad de vida.

\section{Como variable explicatorias se consideraron:}

Condicion oral: El constructo de condición oral se determinó como una sola variable una vez aplicado análisis de factores con base en los diferentes atributos que la constituyen y que fueron: Estado de la dentición (evaluado mediante el índice cariados obturados y perdidos (COP) y el índice de caries radicular (RCI) ). ${ }^{11}$ Estado periodontal (Registrado mediante índice comunitario periodontal de necesidad de tratamiento (ICPTN) y el índice de extensión y severidad (IES). ${ }^{11}$ Estado protésico con base a los criterios establecidos en el Tercer Estudio Nacional de Salud Bucal (ENSAB III). ${ }^{11}$ Flujo salival estimulado de acuerdo a los criterios establecidos por Axelsson y Seif. ${ }^{18,19}$ Funcio- nalidad Oral de acuerdo a los criterios definidos por Mojon. ${ }^{20}$ Higiene Oral que incluye la higiene dental determinados mediante el índice cuantificado de plaCa (ICP) ${ }^{21}$ y el índice que evalúa higiene de prótesis (IEHP) según los criterios definidos por Pietrokovski y colaboradore $\mathrm{s}^{22} \mathrm{y}$ finalmente dolor oral recolectado la mediante Escala Análoga visual.

Condición sistémica: La condición general de salud y la medicación se recolectaron de la historia clínica de acuerdo con los criterios establecidos por Spilker; ${ }^{23}$ en la que se cuantificó la cantidad de condiciones sistémicas presentes y se categorizó según había o no más de dos condiciones sistémicas en un individuo. Para medicación se tuvo en cuenta para la dicotomización el consumo de más de un medicamento. Experiencia de dolor: Para esta variable se consideraron tres atributos: Presencia de dolor, localización e intensidad de dolor, esta última se recolectó mediante una escala análoga visual. Condición sensorial y del lenguaje: Esta condición se adoptó del cuestionario general de salud (GHQ) diseñado por Goldgerg, Crewe y Athelstan; ${ }^{24}$

Condición mental: El nivel cognitivo se determinó aplicando el Examen Mental Abreviado (MINIMENTAL): ${ }^{17,25}$ y la depresión mediante el CES-D. ${ }^{17,26}$ Funcionalidad física: Se evalúo aplicando el Índice de Capacidad Funcional -ICF-17,27 que evalúa los atributos de: movilidad básica, traslado básico, alimentación; aseo personal, vestido, desplazamiento, balance y continencia. El rango total de la escala está entre cero y 100 puntos y el punto de corte 70 puntos.

Valoración social: Definida como la percepción de la relación con las otras personas y el tipo de redes de soporte, se analizó mediante el cuestionario de redes de apoyo y el de redes sociales de la vida adulta. ${ }^{28}$ Las variables sociodemográficas recolectadas fueron: la edad en años cumplidos y la escolaridad como el total de años educativos cursados. Edad se categorizó considerando como punto de corte registrar más de 70 años y educación más de 1 año de escolaridad; además, se recolectaron género, tipo de afiliación al sistema de seguridad social, ocupación o actividad que realiza dentro de la institución geriátrica y vinculación institucional.

Los procedimientos incluyeron la realización de una prueba piloto en la cual la evaluadora, se entrenó y se estandarizaron los criterios de diagnóstico para cada una de las variables contempladas en el formulario. Para el trabajo de campo se realizaron algunas adaptaciones locativas para facilitar la recolección de la información. La colecta de la información se inició con la revisión de la historia clínica del pa- 
ciente y se determinó si registraba algún criterio que lo excluyera del estudio, hecho que se corroboró con el personal de enfermería. Aquel que cumplía con los criterios de inclusión se citaba a la consulta y se procedía al examen previa obtención del consentimiento respectivo.

La recolección de la información se inició con las variables sociodemográficas y posteriormente se aplicaron los cuestionarios relacionados con la salud general, ${ }^{23}$ las redes sociales, ${ }^{28} \mathrm{y}$ la condición mental. ${ }^{17}$ así como de las otras variables incluidas. Para el examen intraoral se procedió a un examen sistemático de las condiciones orales de acuerdo con los parámetros establecidos para cada uno de los indicadores utilizados. ${ }^{11,18,20,21}$ Las medidas de bioseguridad en cada examen oral fueron aplicadas en forma estricta de acuerdo a las normas establecidas. ${ }^{29}$

Una vez recolectada la información se procedió a efectuar la codificación de cada uno de los cuestionarios y a la sistematización por duplicado, en el programa Excel, ${ }^{30}$ la información obtenida se exportó al programa EPI-INFO $6.04^{31}$ y mediante la rutina validate se procedió a verificar la calidad de la digitación de la misma, se efectuaron las correcciones necesarias y se exportó al paquete estadístico STATA $9.1^{32}$ para su correspondiente procesamiento.

Para el análisis de la información, Inicialmente se aplicó un análisis univariado para cada una de las variables incluidas en el estudio, aplicando medidas resumen de tendencia central y dispersión dependiendo de la distribución de la variable. ${ }^{33} \mathrm{El}$ constructo de condición oral y los atributos que lo definen fue evaluado aplicando un análisis de factores. ${ }^{34}$ Finalmente se generó una nueva variable, condición oral, con promedio de cero y varianza uno, base del análisis posterior.

Posteriormente las variables continuas se categorizaron de acuerdo con los criterios establecidos y se realizó un análisis bivariado inicial, en el que se evaluaron asociaciones de la variable salida (calidad de vida) con cada una de las variables explicatorias mediante test de Chi Cuadrado y test Exacto de Fisher. ${ }^{33} \mathrm{La}$ evaluación de posible confusión se determinó mediante un análisis estratificado para cada una de las variables explicatorias y según las asociaciones de la calidad de vida y la condición oral. ${ }^{35,36}$

La elaboración del modelo multivariado para calidad de vida se realizó aplicando regresiones logísticas siguiendo las recomendaciones de Hosmer y Lemeshow. El modelo final incluyó los potenciales confusores que permitieron su ajuste. Una vez seleccionado el modelo, se aplicaron las pruebas de bondad de ajuste de acuerdo con los parámetros establecidos por Greenland, Hosmer y Lemeshow. ${ }^{35}$ Finalmente y considerando que el modelo logístico sobreestima el Odds Ratio (OR) según la prevalencia de la condición evaluada, se establecieron los Riesgos Relativos (Razones de prevalencia) mediante el método de corrección del OR propuesto por Zhang y Yu. ${ }^{37}$ Todo el análisis se realizó considerando un nivel de significancia $\alpha=0.05$.

Para la ejecución de los exámenes, del adulto mayor, se siguieron las normas establecidas en la Resolución 008430 de 1993 del Ministerio de Salud de la República de Colombia, ${ }^{38}$ para trabajos de investigación que involucran seres humanos. Por tratarse de un estudio observacional de corte transversal, en el que el investigador se limitó a examinar y a recolectar la información relacionada con la investigación (de acuerdo con el artículo 11 parágrafo b) se clasifica como investigación de riesgo mínimo. Adicionalmente este trabajo se enmarcó en los principios éticos fundamentales de Beneficencia, autonomía, confidencialidad.

\section{RESULTADOS}

\section{Análisis univariado}

La población de estudio estuvo conformada por 523 personas mayores, vinculados a tres instituciones geriátricas de Bucaramanga y la muestra la constituyeron 106 adultos mayores. Es importante anotar que el total de la muestra requerida para mostrar la posible asociación de interés es de 220 adultos mayores; por lo cual se optó que la mitad de la población se evaluaría en la segunda fase y la otra mitad en fase III de este proyecto; la selección de los sujetos se hizo mediante muestreo aleatorio simple.

Las características sociodemográficas de la población evaluada se resumen en la Tabla 1; cabe resaltar sin embargo, que dentro de los adultos mayores analizados cerca del 7\% de ellos no registraban vinculación a régimen alguno. El promedio de edad en la población encuestada fue de $73 \pm 9.1$, con mediana de 73.5 y un rango entre los 50-92 años. La escolaridad mostró un promedio de $2 \pm 2.7$, con mediana de 1 y rango entre 0 y 11 años.

Al referirse a la condición sistémica se destaca que el $56.6 \%(60)$ registran más de dos afecciones sistémicas (Tabla 2); El promedio de medicamentos suministrados fue de $1.2 \pm 0.87$ y el $38.7 \%$ de la población consume más de un medicamento. 


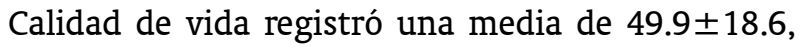
con una mediana de 52 y un rango entre 14 y 85 $\mathrm{mm}$. Al analizar los aspectos relacionados con la condición sensorial y del lenguaje cabe destacar que la condición visual se observó alterada en el 62.3\% (66) de las personas evaluadas, mientras que para audición y lenguaje en el 34,9\%(37) y 30.2\% (32) respectivamente.

Tabla 1. Descripción de las variables sociodemográficas de los adultos mayores vinculados a la Fundación Albeiro Vargas y Ángeles Custodios (FAVAC) durante el segundo semestre de 2004

\begin{tabular}{lcc}
\hline \multicolumn{1}{c}{ Variable } & $\mathbf{n}=\mathbf{1 0 6}$ & $\mathbf{\%}$ \\
\hline $\begin{array}{l}\text { Género } \\
\text { - Femenino }\end{array}$ & 53 & 50 \\
- Masculino & 53 & 50 \\
Vinculación institucional & & \\
- San Rafael & 39 & 36.8 \\
- CBA & 25 & 23.6 \\
- San Antonio & 42 & 39.6 \\
Vinculación régimen de seguridad & & \\
- No & 7 & 6.6 \\
- Si & 99 & 93.4 \\
Realizan actividades en institución & & \\
- No & 34 & 32.1 \\
- Si & 72 & 67.9 \\
Edad (años) & & \\
- $\quad>70$ & 40 & 37.7 \\
- >70 & 66 & 62.3 \\
Escolaridad (años) & & \\
- $\quad 1$ & 47 & 44.3 \\
- $\quad>1$ & 59 & 55.7 \\
\hline *Centro de Bienestar del Anciano de Bucaramanga &
\end{tabular}

Tabla 2. Descripción de la calidad de vida y las condiciones sistémicas en la población vinculada a FAVAC durante el segundo semestre de 2004.

\begin{tabular}{|c|c|c|}
\hline Variable & $\mathrm{n}=106$ & $\%$ \\
\hline \multicolumn{3}{|l|}{ Calidad de vida } \\
\hline - Buena & 52 & 49.1 \\
\hline - $\quad$ Mala & 54 & 50.9 \\
\hline \multicolumn{3}{|l|}{ Dolor } \\
\hline - $\quad$ No & 35 & 33 \\
\hline - $\quad \mathrm{Si}$ & 71 & 67 \\
\hline \multicolumn{3}{|c|}{ Enfermedades sistémicas } \\
\hline - $\quad \leq 2$ & 46 & 43.4 \\
\hline - $\quad>2$ & 60 & 56.6 \\
\hline \multicolumn{3}{|l|}{ Medicación } \\
\hline - $\quad \leq 1$ & 65 & 61.3 \\
\hline - $\quad>1$ & 41 & 38.7 \\
\hline \multicolumn{3}{|l|}{ Afección de los sentidos } \\
\hline - $\quad$ No & 68 & 64.2 \\
\hline - $\quad \mathrm{Si}$ & 38 & 35.8 \\
\hline
\end{tabular}

Las condiciones que registraron mayor prevalencia fueron redes sociales inadecuadas en el 99.1\%(105) y mala condición oral en el $67.9 \%$ (72). A pesar de estar cercana al 50\%, el deterioro físico funcional fue la condición menos frecuente, 48.1\% (31) (Tabla 3).

Atributos relacionados con la condición oral: A nivel dental se hace evidente un promedio alto (23.2) en el índice COP-d (dientes cariados, obturados y perdidos) y el componente de perdidos es el que más le aporta al índice con promedio de 23.1. El flujo salival esta ligeramente disminuido con un promedio de $0.93 \mathrm{mlt} /$ min. La higiene dental y de prótesis dental se pueden catalogar como malas con un promedio de 1.99 y 67 puntos respectivamente (Tabla 4).

Para el análisis de factores que definió la variable condición oral es importante señalar que los atributos seleccionados (COP, dolor oral y flujo salival) tienen un peso importante sobre la variable condición oral, definida en este estudio (Tabla 5). Finalmente el análisis de esta variable, generada a partir del análisis factorial mostró un promedio de cero y una varianza de 1.0 , con un rango entre -1.84 y 3.87 , la cual se categorizó como buena si los valores eran positivos y mala con signo negativo.

Tabla 3. Descripción de las condiciones sociales, mentales, orales y fisicas funcionales en la población vinculada a FAVAC durante el segundo semestre de 2004.

\begin{tabular}{lcc}
\hline \multicolumn{1}{c}{ Variable } & $\mathbf{n = 1 0 6}$ & $\mathbf{\%}$ \\
\hline $\begin{array}{l}\text { Redes de apoyo } \\
\text { - Adecuadas }\end{array}$ & 36 & 34 \\
- Inadecuadas & 70 & 66 \\
Redes sociales & & \\
- Adecuadas & 1 & 0.9 \\
- Inadecuadas & 105 & 99.1 \\
Ces-d & & \\
- Sin depresión & 50 & 47.2 \\
- Depresión & 56 & 52.8 \\
Minimental & & \\
- Normal & 49 & 46.2 \\
- Deteriorado & 57 & 53.8 \\
Capacidad funcional & & \\
- Normal & 55 & 51.9 \\
- Deteriorada & 51 & 48.1 \\
Condición oral & & \\
- Buena & 34 & 32.1 \\
- Mala & 72 & 67.9 \\
\hline
\end{tabular}


Tabla 4. Descripción de la condición dental, periodontal, flujo salival, higiene.

\begin{tabular}{|c|c|c|c|c|}
\hline Variable(n=\#sujetos) & Promedio \pm D.E* & Min-Max & Mediana & RIC** \\
\hline \multicolumn{5}{|l|}{ Condición dental(n=106) } \\
\hline - $\mathrm{COP}$ & $23.2 \pm 7.3$ & $0-28$ & 27 & $21-28$ \\
\hline C & $0.4 \pm 1.1$ & $0-7$ & 0 & $0-0$ \\
\hline $\mathrm{o}$ & $0.3 \pm 1.3$ & $0-10$ & 0 & $0-0$ \\
\hline $\mathrm{P}$ & $23.1 \pm 7.2$ & $0-28$ & 26.5 & $21-28$ \\
\hline - $\quad \operatorname{RCI}(\mathrm{n}=106)$ & $0.21 \pm 1.27$ & $0-10$ & 0 & $0-0$ \\
\hline Flujo Salival(n=103) & $0.93 \pm 0.22$ & $0.1-1.5$ & 1 & $0.8-1.1$ \\
\hline Higiene dental $(\mathrm{n}=96)$ & $1.99 \pm 0.57$ & $0.66-3$ & 2 & $1.7-2.3$ \\
\hline Higiene de prótesis $(n=63)$ & $67 \pm 30$ & $0-100$ & 66 & $50-83$ \\
\hline Dolor oral $(n=68)$ & $5.8 \pm 14.1$ & $0-57$ & 0 & $0-0$ \\
\hline
\end{tabular}

Dolor oral $(\mathrm{n}=68)$

$5.8 \pm 14.1$

$0-57$

Tabla 5. Coeficiente de los puntajes de las variables incluidas en el factor seleccionado.

\begin{tabular}{llc}
\hline & \multicolumn{1}{c}{ Atributos } & Coeficiente de los puntajes \\
\hline - & Cariados & 0.45706 \\
- Obturados & 0.25242 \\
- Perdidos & -0.53708 \\
- Dolor oral & 0.19503 \\
- Flujo Salival & 0.18202 \\
\hline
\end{tabular}

\section{Análisis bivariado}

Las variables labor, consumo de medicamentos, depresión y capacidad fisica funcional afectada registraron asociaciones estadísticamente significativas con la calidad de vida. (Tabla 6). La exploración inicial de los Odds Ratio (OR) crudos se muestra en la Tabla 6 y en la que se hace evidente la asociación positiva de

enfermedades sistémicas, consumo de medicamentos, depresión y discapacidad física funcional con una mala calidad de vida. Estas variables son la base del modelo multivariado. Al analizar la asociación de la condición oral con la calidad de vida estratificada por cada una de las variables en estudio, no se observó que con alguna de ellas existiese confusión.

\section{Análisis multivariado}

El modelo multivariado de regresión logística ajustado (Tabla 7) no se evidencia una potencial asociación entre la condición oral y la calidad de vida después de ajustar por capacidad física funcional, edad y consumo de medicamentos. Se resalta además, el evidente efecto de sobreestimación de los Odds Ratio en relación con los RR para este modelo, como se esperaba.

Tabla 6. Variables evaluadas en la población adulta mayor asociadas con la calidad de vida.

\begin{tabular}{lcccccc}
\hline \multicolumn{1}{c}{ Variables } & Global & \multicolumn{2}{c}{ Calidad de vida } & & & \\
& & Mala & Buena & & & \\
& $\mathrm{n}=106$ & $\mathrm{~N}=54$ & $\mathrm{~N}=52$ & ORcrudo & IC95\% & $\mathbf{P}^{*}$ \\
& $\mathrm{Frec}(\%)$ & $\mathrm{Frec}(\%)$ & $\mathrm{Frec}(\%)$ & & & \\
\hline Labor & & & & & & \\
$\mathrm{Si}$ & $72(67.9)$ & $30(41.7)$ & $42(58.3)$ & 0.29 & $(0.12-0.71)$ & 0.007 \\
No & $34(32.1)$ & $24(70.6)$ & $10(29.4)$ & & & \\
Enfermedades sistémicas & & & & & \\
$>2$ & $60(56.6)$ & $35(58.3)$ & $25(41.7)$ & 1.98 & $(0.9-4.3)$ & 0.084 \\
$\leq 2$ & $46(43.4)$ & $19(41.3)$ & $27(58.7)$ & & & \\
Medicamentos & & & & & \\
$>1$ & $41(38.7)$ & $27(65.9)$ & $14(34.1)$ & 2.71 & $(1.2-6.1)$ & 0.016 \\
$\leq 1$ & $65(61.3)$ & $27(41.5)$ & $38(58.5)$ & & & \\
CESD & $56(52.8)$ & $35(62.5)$ & $21(37.5)$ & 2.72 & $(1.2-6.0)$ & 0.013 \\
Depresión & $50(47.2)$ & $19(38)$ & $31(62)$ & & & \\
Sin depresión & & & & & \\
Capacidad funcional & $51(48.1)$ & $32(62.8)$ & $19(37.2)$ & 2.52 & $(1.15-5.5)$ & 0.020 \\
Deteriorada & $55(51.9)$ & $22(40)$ & $33(60)$ & & & \\
Normal & & & & & \\
Condición oral & $72(67.9)$ & $35(48.6)$ & $37(51.4)$ & 0.74 & $(0.3-1.7)$ & 0.485 \\
Mala & $34(32.1)$ & $19(55.9)$ & $15(44.1)$ & & & \\
Buena & & &
\end{tabular}

*Test de Chi cuadrado ${ }^{* *}$ Test exacto de Fisher

80 
Tabla 7. Modelo de regresión logística ajustado de la calidad de vida y la corrección de los respectivos riesgos relativos.

\begin{tabular}{|c|c|c|c|c|}
\hline Variable & $\mathbf{O R}^{*}$ & IC95\% & $\mathbf{R R}^{* *}$ & IC95\% \\
\hline Capacidad funcional & 2.62 & $1.02-6.7$ & 1.6 & $1.01-2.14$ \\
\hline Edad & 1.08 & $1.03 ; 1.14$ & -- & --- \\
\hline Medicamentos & 0.71 & $0.42 ; 1.2$ & -- & -- \\
\hline Condición oral alterada & 0.97 & $0.4 ; 2.5$ & 0.99 & $0.38 ; 2$ \\
\hline
\end{tabular}

*Odds Ratio ** Razón de prevalencia

Las pruebas de diagnóstico de la bondad de ajuste de la regresión para este modelo mostraron un $\chi^{2}$ Pearson de $66(\mathrm{p}=0.28)$ y $\chi^{2}$ HL de $7.69(\mathrm{p}=0.46)$ lo que indica un buen ajuste del modelo logístico. Así mismo, los residuales presentan un promedio y una varianza cercanos a cero y a uno respectivamente, apoyando los hallazgos anteriores.

Complementariamente, el análisis gráfico del diagnóstico de la regresión se muestra en la figura 1; en las que se aprecia en general una distribución de probabilidades entre 0 y 1 y es evidente un buen ajuste del modelo.

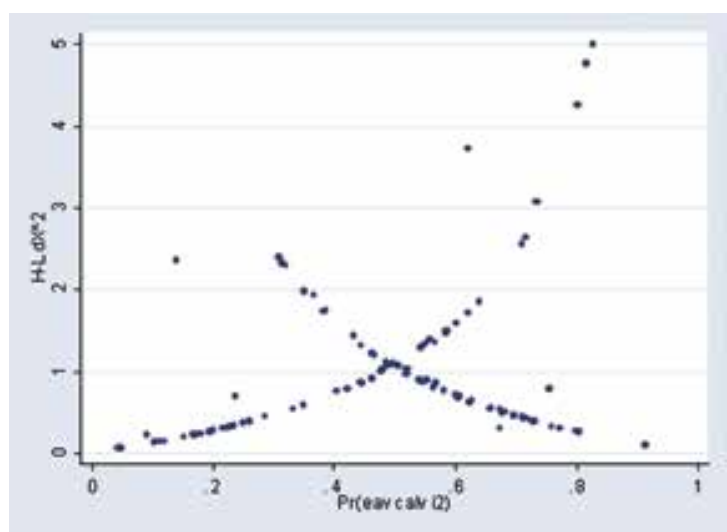

Figura 1. Diagrama de dx2 Vs Probabilidad logística estimada para el modelo de calidad de vida.

\section{DISCUSIÓN}

El objetivo de la presente investigación fue evaluar la asociación de la condición oral con la calidad de vida en las personas mayores vinculadas a instituciones geriátricas, por lo cual es importante retomar los resultados y analizar sus posibles implicaciones en la atención integral que se brinda a la persona mayor de estas instituciones.
No es fácil comparar los hallazgos relacionados con la calidad vida en este trabajo con los resultados de investigaciones previas; en primer lugar porque el auge de la investigación en este parámetro, se ha centrado en la validación de instrumentos e índices que la evalúen y en segundo lugar, son muy variados los mecanismos implementados para su análisis. ${ }^{7,17,39}$

Jiménez-Férez y colaboradores, ${ }^{40}$ encontraron en México una mejor calidad de vida en un grupo similar con un $84 \%$ de los adultos mayores con puntajes entre 80 y 100 y sólo el 11\% la calificó con puntajes inferiores a 60. En las instituciones geriátricas involucradas, sólo el 3.8\% de la población registró puntajes entre 80 y 100 y el $62.3 \%$ reportó valores inferiores a 60, lo que demuestra una mejor percepción en la población mexicana al compararse con la de este estudio; las diferencias podría sustentarse en que en esta última, la población evaluada es institucionalizada, con mayor dependencia física, económica y social, aspectos estrechamente relacionados con calidad de vida. ${ }^{17}$

A nivel periodontal es importante anotar que el $85.7 \%$ de la población en la que se pudo evaluar esta condición (36/42) registraba algún marcador periodontal, comparado con el $96 \%$ y el $94 \%$ de los registrados para personas mayores de 55 años en el ENSAB III y en el estudio de Bucaramanga respectivamente. El promedio de COP en los adultos mayores de vinculados a instituciones geriátricas fue de 23.2 relativamente similar al registrado por otra población mayor de Bucaramanga y mayor al reportado en el grupo de personas mayores del Estudio Nacional de Salud Bucal (19.7); en los cuales, el componente de dientes perdidos determinó la magnitud del deterioro para este atributo. ${ }^{11,13} \mathrm{En}$ conclusión la población mayor en Colombia pierde sus dientes, lo cual incide en su funcionalidad oral, que se vio afectada en el $61.8 \%$ de los sujetos en este trabajo. 
En el mundo la prevalencia de edentulismo oscila entre el 6 y $69 \%,{ }^{41}$ en Colombia es del $65 \%,{ }^{11}$ en la población mayor institucionalizada de Bucaramanga analizada en el 2003 se encontró en el 55\% ${ }^{13}$, cercana a la detectada en este trabajo con $54 \%$ y a la registrada en países como Malasia (57\%) y Bulgaria (53\%), lo que parece indicar que la pérdida dentaria es un patrón característico de las personas mayores a nivel mundial y no sólo en Colombia, lo que sugiere también algún nivel de compromiso en su capacidad oral funcional. ${ }^{41}$

Es importante recordar que en el adulto mayor se registran múltiples cambios físicos y funcionales, que se manifiestan frecuentemente como patologías, sin que exista claridad entre lo normal y anormal. ${ }^{5,6}$ Se ha demostrado, una mayor prevalencia de enfermedades cardiovasculares, pulmonares, renales, hepáticas, gastrointestinales, músculo-esqueléticas, etc; como efecto primario o secundario del deterioro relacionado con el envejecimiento. ${ }^{42}$

La población adulta mayor vinculada a a instituciones geriátricas en el presente trabajo no fue ajena a este hecho, se destaca que el $75.5 \% \%$ de las personas evaluadas reportaban recibir algún tipo de medicación. En cuanto al promedio de sistemas involucrados, se estableció en esta investigación un promedio 2.48 y 1.2 medicamentos consumidos ( $38.7 \%$ consumían más de un medicamento). Estas cifras son inferiores a las reportadas por Locker y Gibson $^{43}$ en población mayor de Canadá, pues en promedio las personas registraban 4.5 condiciones sistémicas y el $93 \%$ les administraban más de un medicamento; la posible diferencia entre las dos poblaciones se le podría atribuir a un promedio de edad mayor (83.3 años) en los canadienses, lo cual esta a favor del incremento concomitante de enfermedades sistémicas con la edad.

La depresión es una condición que aqueja a la sociedad de hoy y particularmente a las personas mayores. En Bucaramanga un estudio previo realizado con población institucionalizada registró que el 55.3\% de la población presentaba depresión leve o moderada, ${ }^{13}$ cifra similar ala detectada $(52.8 \%)$, lo que podría considerarse alto, por lo cual debe ser evaluada y considerada en el momento de desarrollar cualquier tipo de intervención con este grupo de personas.
La capacidad física funcional como efecto del deterioro fisiológico es evidente en las personas mayores; en un estudio realizado en ancianos de Manizales y en el que se analizaba la capacidad de realizar las Actividades Básicas Cotidianas (mediante escala de Barthel) indicó que el 35.2\% de las personas mayores registraban algún nivel de dependencia y en la población mayor de este trabajo fue de $48.1 \%$; su importancia radica en la necesidad de no subestimar esta condición, que se hace más evidente con el aumento de la edad y que limita la capacidad de las personas mayores de realizar las actividades más elementales y necesarias para la vida, como el aseo personal, el vestido y desplazamiento. ${ }^{44}$

La asociación de la una mala condición oral con una calidad de vida deteriorada registró un RR.. cercano a 1 (IC95\% 0.38-2.0), lo cual parece indicar que la salud oral no incide sobre la calidad de vida en la población evaluada. Múltiples estudios se han realizado para establecer la asociación la condición oral con la calidad de vida; ${ }^{45-47}$ dentro de los que cabría citar a Locker, Clarke y Payne, ${ }^{45}$ quienes, lograron establecer una asociación entre una mala condición oral con malestar psicológico y con bajos niveles de satisfacción frente a la vida en las personas mayores; aspectos inherentes al concepto de calidad de vida y que son contrarios a los resultados presentados. Esta asociación también fue detectada por McGrath y Bedi, ${ }^{46}$ quienes lograron establecer un riesgo 1.32 veces mayor de tener mala calidad de vida en personas mayores con alteraciones bucodentales al compararse con aquellos que no las presentaban. Steele y colaboradores ${ }^{47}$ exploraron la posible relación de la pérdida dental con la calidad de vida y sugieren que el impacto de la salud bucal sobre esta, se reduce con el incremento de la edad y esto podría explicar los resultados en el presente estudio.

Es importe considerar el contexto en el que las personas mayores viven; pues la cultura, la procedencia y aun el estrato socioeconómico, puede llegar a alterar la percepción sobre su condición oral y modificar la relación de la salud bucodental con la calidad de vida; hecho que podría evidenciarse en el presente estudio, pues la mayoría de las personas entrevistadas pertenecían a cohortes en las que la pérdida dentaria era un procedimiento mutilante rutinario; por lo cual su percepción de la salud oral y el impacto sobre su vida podría ser un aspecto subvalorado. ${ }^{47}$ 
El impacto negativo de una mala condición oral sobre la calidad de vida y la salud es particularmente significativa entre las personas edéntulas. La pérdida extensiva de dientes reduce la capacidad masticatoria y afecta la elección de los alimentos, por lo cual las personas desdentadas evitan los alimentos fibrosos y prefieren aquellos ricos en grasas saturadas y colesterol, lo cual genera otro tipo de problemas como obesidad, alteraciones cardiovasculares y músculo esqueléticas, entre otras. ${ }^{48}$

La relación entre la discapacidad física con la calidad de vida ha sido estudiada, ${ }^{17,44}$ encontrando por ejemplo que el $60 \%$ de las personas que presentaban discapacidades fisicas consideraban que tenían comprometida su calidad de vida, ${ }^{44}$ cifras similares a las encontradas en el presente estudio en el que el $62.8 \%$ de las personas con capacidad física deteriorada registraban tener mala calidad de vida.

Es importante clarificar sobre las posibles limitaciones de este trabajo. Esta investigación se concibió originalmente como un estudio que buscaba abordar a los adultos mayores institucionalizados de Bucaramanga y su área metropolitana, por lo cual la muestra inicial calculada era de aproximadamente 220 adultos mayores.

Por razones inherentes al proceso investigativo, se hizo necesario desarrollar este trabajo sobre una parte de la población mayor vinculada a instituciones geriátricas de tres instituciones que aceptaron vincularse a este proceso, hecho que no permitió el control del sesgo de selección y le resto poder al estudio. ${ }^{49}$

Sin embargo y considerando estudios anteriores relacionados con población institucionalizada de Bucaramanga y su área metropolitana, ${ }^{12,13}$ se podría pensar que las personadas evaluadas en la presente investigación, en términos generales no difiere significativamente de los adultos mayores residentes en otros hogares geriátricos de la ciudad. En dicho estudio, que evalúo 284 personas mayores de 12 hogares geriátricos de la ciudad. Mostró como el promedio de edad $77.8 \pm 8.8$ años, similar al de la población de la Fundación, $75 \pm 8.4$ años.

En cuanto a la vinculación al régimen subsidiado fue mayor en este estudio $93.4 \%$ comparado con $40 \%$ del citado autor; esto probablemente se explica por una mayor cobertura en seguridad social, política que actualmente se ha hecho más efectiva para la población adulta mayor institucionalizada. El nivel de analfabetismo es similar al reportado en otros estudios, al igual que los antecedentes clínicos de orden sistémico como la hipertensión y la medicación con antisicóticos. ${ }^{12}$

Al considerar atributos relacionados con la condición oral, es importante anotar que en el trabajo desarrollado por Bermúdez y colaboradores, y García y colaboradores tanto la condición periodontal como la higiene oral registran un comportamiento similar al observado en la población adulta mayor examinada en este trabajo y que pueden catalogarse como comprometidas. ${ }^{12,50}$

En esta investigación una buena condición oral se definió como la oportunidad que provee el sistema estomatognático para vivir una vida satisfactoria, pues aporta las condiciones biológicas, funcionales y estéticas que le permiten al individuo relacionarse adecuadamente consigo mismo y con los otros, desarrollar una vida plena de acuerdo a sus expectativas, alcanzar y mantener sus condiciones de salud general dentro de su propio contexto; por lo tanto los atributos definidos y que fueron seleccionados deben analizarse a la luz de la literatura que los soporta.

Como resultado del análisis de factores el índice COP (dientes cariados obturados y perdidos), flujo salival y dolor oral se constituyeron en los atributos que permiten definir la condición bucodental de los adultos mayores de las tres instituciones geriátricas involucradas; algunos autores sostienen que el COP y particularmente el componente de perdidos, es un aspecto clave y que coincide con los resultados observados en el trabajo realizado con las personas mayores institucionalizados, pues fue uno de los atributos con mayor carga (-0.53)..$^{9,11,51}$

La inclusión de este atributo se sustenta adicionalmente, en su impacto sobre la autoestima, puesto que los dientes se constituyen en uno de los aspectos más importantes de la imagen que se proyecta socialmente y los individuos conciente o inconscientemente buscan la aprobación social para mantener una autoevaluación positiva. En términos funcionales la presencia de dientes permite la masticación y fonación adecuada, además de promover la expresión de sentimientos. ${ }^{51}$ 
El segundo atributo incluido fue el flujo salival con una carga de 0.18 en el análisis de factores. Su inclusión se fundamenta en el rol esencial que cumple la saliva en la preservación de la homeostasis de cavidad oral. Aspecto frecuentemente afectado durante el proceso de envejecimiento y como efecto secundario a la medicación, a la que los adultos mayores se ven sometidos dadas sus condiciones sistémicas crónicas. ${ }^{52,53}$

La reducción del flujo salival afecta la capacidad masticatoria, la elección de los alimentos, el poder degustar y disfrutarlos, por lo cual las personas dejan con frecuencia de comer; con repercusión sobre el estado nutricional y la condición general. Por lo cual es importante su valoración regular en el análisis de la condición oral del individuo mayor. ${ }^{54}$

El último atributo incluido fue dolor oral y su carga fue de 0.19 . Al referirse a este, es importante destacar el efecto limitante que tiene sobre la ejecución de actividades orales diarias como es masticar, beber, hablar y besar; adicionalmente, el dolor en general y específicamente el oral, se ha relacionado con ansiedad y depresión, incidiendo sobre la salud y la calidad de vida de los ancianos; usualmente las personas mayores relacionan dolor oral con enfermedad bucodental. Pues el "dolor de muela" es una experiencia muy desagradable que permanece grabada en su memoria. ${ }^{9}$

A pesar de estos hallazgos es importante señalar que el constructo definido inicialmente, no incluyó otros atributos asociados con la condición oral en este grupo etáreo y que son reportados en la literatura, ${ }^{11,14}$ tales como ICPTN, IES, ICP y estado de prótesis que no pudieron ser incluidos en el análisis de factores puesto que el tamaño de muestra al incluir a cada uno de ellos se reducía hasta en un $70 \%$ y hacía imposible su inclusión. Es importante señalar que estos hallazgos son un llamado para la profesión odontológica, en el sentido de generar y validar nuevos indicadores de la condición oral en adultos mayores institucionalizados, dado que sus condiciones son particularmente deficientes y diferentes al resto de la población.

Como fortalezas de este trabajo cabría destacar, la valoración integral realizada, aspecto señalado por varios autores como una necesidad vital para la toma de decisiones de intervención, tanto terapéutica como preventiva en la población adulta mayor.
Los resultados referidos ilustran la necesidad de una visión y una atención integral del adulto mayor por la multiplicidad de factores y de los efectos que la salud general y oral podría tener sobre la calidad de vida y el bienestar. ${ }^{4}$

La información obtenida no permite sugerir asociación de la condición oral con la calidad de vida y pero parece mostrar como ilustrar sobre las construcciones que los adultos mayores han hecho alrededor de su salud oral, lo que favorece la orientación de acciones dirigidas a contribuir sobre la salud bucodental y sobre la calidad de vida de estos adultos mayores; puesto que las acciones de todo programa para la promoción de la salud y prevención de las enfermedad, así como los orientados a modificar perfiles epidemiológicos, deben partir de la identificación de las necesidades particulares en cada grupo poblacional, los factores y los grupos de riesgo. ${ }^{55}$

De otro lado, la aplicación de índices e instrumentos validados y con los cuales se realizó la estandarización, confiere consistencia a los hallazgos presentados y se constituye en uno de los principales fundamentos que soporta la ausencia de un sesgo de clasificación. ${ }^{5}$

Se concluye que una condición física funcional deteriorada es un mejor predictor de la mala calidad de vida en las personas mayores y que la condición oral e impacto sobre la vida de las personas mayores podría estar afectada por aspectos culturales que hacen que la sociedad en general y el adulto mayor en particular, pueda llegar a subestimar esta condición

Es importante señalar, que este artículo presenta sólo los resultados encontrados en la segunda fase de recolección de datos y que aun no hay poder suficiente que permita suportar las conclusiones planteadas; Los resultados que reflejan las tres fases del proceso se espera, puedan presentarse en un nueva publicación una vez se culmine con la tercera fase de este proceso, que a la fecha se está desarrollando.

\section{AGRADECIMIENTO}

Las autoras agradecen a la Universidad Santo Tomás por el apoyo financiero brindado al presente proyecto. 


\section{BIBLIOGRAFÍA}

1. Naciones unidas. Comisión para el desarrollo social. Estrategia Internacional para la Acción 2002 para el Envejecimiento. 29 de agosto de 2001. p 1-3

2. Colombia joven. Hacía una política pública de juventud en Colombia. Análisis situacional de la juventud colombiana en el naciente milenio.[online] p. 10-15. URL disponible en: http://www.colombiajoven.gov.co/documentos/ gemerales/hu2.pdf

3. Ham-chande R. El envejecimiento: Una nueva dimensión de la salud en México. Salud Pública de México. 1996; 38: 409-418

4. Goldberg TH, Chavin SI. Preventive medicine and screening in older adults. J Am Geriatric Soc 1997; 45: $344-$ 354.

5. Wallance RB, Woolson RF. The epidemiologic study of elderly. Oxford: University press. 1992. p. 9,163 - 165, 196 -212 .

6. Fried LP. Epidemiology of ageing. Epidemiologic Rev 2000; 22: 95 - 106.

7. Velarde-Jurado E, Avila-Figueroa C. Consideraciones metodológicas para evaluar la calidad de vida. Salud Pública de México 2002; 44: 448 - 463.

8. Lugo LH, García HI, Gómez C. Calidad de vida y calidad de vida relacionada con salud. IATREIA 2002; 15: 96 - 102.

9. Marin DJ. Influencia de la boca en la calidad de vida de los ancianos. Revista de la Asociación Colombiana de Gerontología y Geriatria 2002; 16: 363 - 368.

10. Instituto Nacional de Salud. La ancianidad: Proyecto de la tercera edad. En: Encuesta Nacional de Conocimientos, Actitudes y Prácticas en Salud. Colombia 1986-1989. Santafé de Bogotá: Ministerio de Salud, División de Investigaciones Especiales, 1991 p.1 - 83.

11. Ministerio de Salud. Estudio Nacional de Salud Bucal-ENSAB III. Bogotá, Colombia, 1999. Tomo VII, p. 9-16,18-26,36-38 p.99

12. Bermúdez W. Concha SC, Camargo DM. Perfil orofacial de las personas mayores institucionalizadas de la ciudad de Bucaramanga y su área metropolitana. Ustasalud Odontología 2003; 2: 13 - 19.

13. Espindola G. Pinzón AR, Daza CR. Concha SC y Camargo DM. Asociación entre el estado mental y la condición oral de la persona adulta mayor institucionalizada de la ciudad de Bucaramanga y su área metropolitana. Ustasalud Odontología 2003; 2: 31 - 32

14. Department of Health and Human Services of United States. Oral Health in America: In: Report of the Surgeon General. Rockville, MD. Department of Health and Human Services. U.S. Public Health Service. National Institute of Dental and Craniofacial Research. National Institute of Health, (2000) Chapter 5 and 6

15. Fenstein J, Alvan R. Clinical Bioestatistics. The architecture of cross-sectional research (part I). Clinic Pharmacol Ther 1978; 81 - 96.

16. Fenstein J, Alvan R. Clinical Bioestatistics. The architecture of cross-sectional research (Conclusion). Clinic Pharmacol Ther 1978; 481 - 493.

17. Orozco MV, Orozco LC, Herrera JP, Méndez G, Mendoza MY, Pinzón LI, Sánchez VJ. Validación de un instrumento para medir la calidad de vida en sus aspectos fisicos, emocional, cognitivo y social en personas ancianas. Revista de la Asociación Colombiana de Gerontología y Ge- riatria 2004; 18: $606-612$.

18. Axelsson P. Diagnosis and Risk Prediction of Dental Caries. NY: Quintessence Publishing. Vol. 2 (2000) p.133 - 134.

19. Seif Thomas. Cariología. Prevención, diagnóstico y tratamiento contemporáneo de la caries dental. Caracas: Actualidades Medico-Odontológicas Latinoamérica, 1997 p. 236

20. Mojon P, Budtz-Jorgensen EI, Rapin CH. Relationship between oral health and nutrition in very old people. Age and Ageing 1999; 28: $463-468$.

21. Pieschacón MP. Programa piloto de odontología preventiva con escolares del municipio de Bucaramanga. [Trabajo de Grado] Universidad Autónoma de Chile. Facultad de Salud Pública, Santiago de Chile: 1998. p. 2 - 43.

22. Pietrokovski J, Harfin J, Mostavoy R, Levi F. Oral finding in elderly nursing home residents in selected countries: oral hygienic conditions and plaque accumulation on denture surfaces. J Prosthet Dent 1995; 73: 136 - 141.

23. Spilker Bert. Data collections in clinical trials. NY: Raven press, 1991 p.102

24. McDowell I, Newell C. Measuring Health: A guide to rating scales and questionnaires. NY: Oxford University Press. $2^{a}$ Ed. (1996) p. 45

25. Fraser GE, Sing PN, Benneth $H$. Variables associated with cognitive function in elderly California seventh-day Adventists. Am J Epidemiol 1996; 143: 1181 - 1190.

26. Mulsant B, Ganguli M, Seaberg E. The relationship between self-rated health and depressive symptoms in an epidemiological sample of community-dwelling older adults. J Am Geriatr Soc 1997; 45: 954 - 958.

27. Rivera JJ, Camargo DM, Aguirre CL, Camargo NY, Castro MI, Chiquillo ML. Mantilla ML, Niño EL, Novoa AC, Quintero SY. Sistema de evaluación para medir la capacidad funcional en la realización de las actividades básicas cotidianas en individuos con deficiencias neurológicas, neuromusculares y osteomusculares. Salud UIS 1998; 29: $23-31$.

28. Pennix BWJH, Tillburg TV, Kriesgsman DMW, Deg DJH, Boeke AJP, Van Eijk JTM. Effects of social support and personal coping resources on mortality in older age: The longitudinal aging study Amesterdam. Am J Epidemiol 1997; 146: $510-519$.

29. Instituto de Seguros Sociales. Bioseguridad Odontológica. Bogotá. Ed. Géminis, 1998 p.15-33

30. Microsoft Excel 5.0. NY. Microsoft Corporation, 1997

31. CDC-OMS. Epi-info 6, Versión 6.04. Atlanta, (Nov 1996).

32. Stata Corp. Stata Statistical Software. Release 8.0. College station. TX: Stata corporation 2003.

33. Pagano M, Gauvreau K. Principles of Bioestatistics. Belmont: Duxbury Press. (1995) p. 35 - 44, 257 - 263.

34. Norman GR and Streiner DL. Bioestadística. Madrid:Mosby/ Doyma Libros, 1996. p. 129 - 142.

35. Hosmer DW, Lemeshow S. Applied logistic regression. NY: A Wiley-Interscience Publications. John Wiley \& Sons 2000 p. $1-187$.

36. Kleinbaum DG, Kupper LL, Morgenstern H. Epidemiologic Research: Principles and Quantitative Methods. NY: Van Nostrand Reinhold Company, 1982 p. 242 - 265.

37. Zhang J, Yu KF. What's the relative risk? A method of correcting the odds ratio in cohort studies of common outcomes. JAMA 1998; 280: 1690 - 1691. 
38. República de Colombia. Ministerio de Salud. Resolución No. 008430 (Oct 4 1993) Bogotá

39. Staquet MJ, Hays RD, Fayers PM. Quality of life assessment in clinical trials. Methods and Practice. Oxford: University Press. 1998 p. $191-194$.

40. Jiménez J, Esquivel I, Gonzalez-Celis AL. La percepción de la salud bucal como media de calidad de vida en ancianos. Revista de la Asociación Dental Mexicana 2003; 10: $19-24$.

41. Petersen PE, Yamamoto T. Improving the oral health of older people: the approach of the WHO Global Oral Health Program. Community Dent Oral Epidemiol 2005; 33: $81-92$.

42. Shimazaky Y, Soh I, Saito T, Yamashita Y, Koga T, Miyazaky $\mathrm{H}$, Takehara $\mathrm{T}$. Influence of dentition status on physical disability, mental impairment and mortality in institutionalized elderly people. J Dent Res 2001; 80: 340 - 345.

43. Locker D, Gibson B. Discrepancies between self-ratings of and satisfaction with oral health in two older adult populations. Community Dent Oral Epidemiol 2005; 33: $280-288$.

44. Gómez JF, Cursio CL. Autopercepción de salud, presencia de enfermedades y discapacidades en ancianos de Manizales. Revista de la Asociación Colombiana de Geriatría 2004; 18: 706 - 714 .

45. Locker D, Clarke M, Payne B. Self-perceveid oral health status, psychological well-being and satisfaction in older adults population. J Dent Res 2000; 79: 970 - 975.

46. McGrath C, Bedi R. Population based norming of the UK oral health related quality of life measure (QHQoL-UK). Br Dent J 2002; 193: 521 - 524.

47. Steele J, Sanders AE, Slade GD, Allen PF, Lanti S, Nutall $\mathrm{N}$, Spencer AJ. How do age and tooth loss affected oral health impacts and quality of life? A study comparing two nationals' samples. Community Dent Oral Epidemiol 2004; 32: 107 - 114 .
48. Sheiham A, Steele JG, Marcenes W, Lowe C, Finch S, Bates CJ, Prentice A, Walls WG. The relationship among dental status, nutrient intake, and nutritional status in older people. J Dent Res 2001; 80: 408 - 413.

49. Lohr SL. Muestreo: Diseño y análisis. México: Internacional Thompson Editores. 2000 p. $4-21$.

50. García Y, Flórez L, Silva L, Aguilar E, Concha SC. Evaluación de la efectividad de un programa educativo en higiene oral dirigido a cuidadores de adultos mayores de los Asilos San Antonio y San Rafael de Bucaramanga. Ustasalud Odontología 2006; 5: 40 - 48 .

51. Jaramillo G, Botero C, David AM, Hincapié SB. Ramírez NX, Varela JH. Valoración significativa del componente bucal de la salud. Revista Facultad de Odontología Universidad de Antioquia. 2003; 14: 61 - 69.

52. Bergdahl $\mathrm{M}$, Bergdahl J. Low unstimulated salivary flow and subjective oral dryness: Association with medication, anxiety, depression, and stress. J Dent Res 2000; 79: $1652-1658$.

53. Rindal DB, Rush WA, Peters D, Maupomé G. Antidepressant xerogenic medications and restoration rates. Community Dent Oral Epidemiol 2005; 33: $74-80$.

54. Dormenval V, Budtz-Jorgensen E, Mojon P, Bruéyere A. Association between malnutrition, poor general health and oral dryness in hospitalized elderly patients. Age and Ageing 1998; 27: 123 - 128.

55. Munevar AM, Rojas JK, Marín DJ. Perfil epidemiológico bucal de los pacientes de 55 años y más que asisten a las clínicas del geronte a la Facultad de Odontología de la U.N. durante el primer semestre del año 2000 y cual es la percepción que tienen estos pacientes sobre su propio estado de salud bucal. Revista de la Federación Odontológica Colombiana 2001; $7-31$. 